\title{
SOURCE WAVE FORMS OF THREE EARTHQUAKES
}

\author{
By Hugo Beniofa
}

\begin{abstract}
Strain seismograms of the Montana shallow earthquake of August 17, 1959 recorded at Isabella, California have the wave pattern predicted in 1904 by Lamb for a surface pressure pulse. This is equivalent to a dipole source such as given by the vertical bilateral fault slip which was observed at the surface. Similar recordings made at Naña of the deep Peruvian earthquakes of August 19 and 30,1961, $(\Delta=600 \pm \mathrm{km}, h=600 \pm \mathrm{km})$ have the pattern calculated by Pekeris for a buried vertical downward force in the form of a step in time. It thus appears that these shocks were generated by a sudden volume contraction at the focus which could be the result of a sudden change of state.

Failure of conventional seismographs to record the patterns calculated by Lamb and by Pekeris is due to their low sensitivity to the very long period ground movement components involved, and their relatively high sensitivity to the short period components which are rendered oscillatory by the departure of the crustal characteristics from the homogeneous half space assumed in the theoretical computations.
\end{abstract}

\section{INTRODUCTION}

Lamb (1904) calculated the theoretical form of the surface radial displacementtime pattern produced by the application of a vertical impulse at a point in the surface of a homogeneous half-space. The pattern is shown in figure 1. The first pulse represents the $P$ (longitudinal body) wave, the second $S$ (transverse body) wave and the third the Rayleigh wave. To the writer's knowledge this pattern has not been observed on earthquake seismograms hitherto although it has been produced in ultrasonic model seismometry (S. Kaufman and W. L. Roever, 1951), (Howard Tatel, 1954). In this paper recordings of the strain seismographs at Isabella, California and Naña, Peru are presented showing the approximate patterns predicted by Lamb for a shallow vertical impulse source and by Pekeris for a buried vertical step source.

Now that the Lamb pattern has been observed it is not difficult to account for its absence on earlier recordings. The earth departs substantially from the uniform halfspace posited by Lamb. The upper portions are layered, and velocities and elastic and density characteristics vary with depth and with azimuth. As a result of these departures from uniformity, the components of a pulse having wavelengths less than the dimensions of the inhomogeneities, are reflected, refracted, and transformed during propagation in such a way as to produce the complex oscillatory wave train observed on conventional seismograms.

In small earthquakes the duration of faulting is short and the source pulse contains principally high frequency components which produce oscillatory movements only. On the other hand, in large earthquakes the pulse duration is sufficient to include substantial frequency components with wavelengths long in comparison with the crustal inhomogeneities. For these the medium conforms reasonably well to Lamb's condition and consequently they produce wave patterns which approach the Lamb form fairly closely. The periods involved range from about 0.5 minute upwards. Thus, the wave patterns for large shocks are composed of two parts-one 
made up of the short period components which produce oscillatory wave forms, characteristic of the layered inhomogeneous path between source and seismograph, and the second part composed of the long period components for which the earth appears as a homogeneous halfspace of mean characteristics. A simple fault origin such as assumed in the elastic rebound theory is equivalent to a dipole and for long period waves it radiates approximately as an impulse source.

In order to record the Lamb pattern, the earthquake spectrum must contain long period components and must be observed at epicentral distances of not more than about $1500 \mathrm{~km}$, the seismograph must be effective for waves in the period range 1-20 minutes or longer, and its frequency response characteristic must be such as to attenuate components higher than about 0.2 cycle per minute. These instrumental conditions can be met with the strain seismograph (Benioff 1959), operating with a low-pass or band-pass filter.

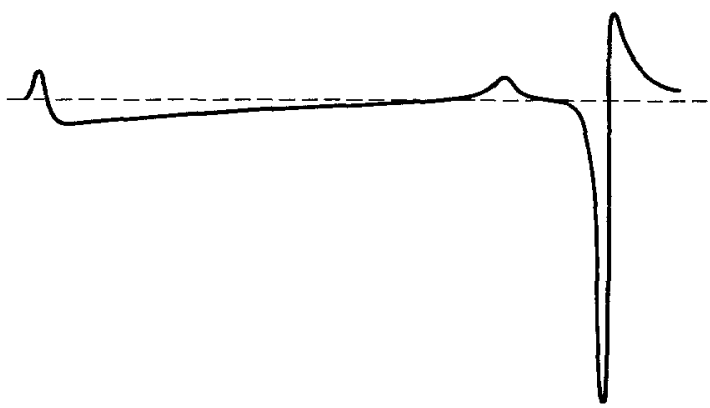

FIg. 1. Surface radial ground displacement pattern produced by vertical downward surface pulse as calculated by Lamb.

The Montana Earthquake of August 18, 1959

Figure 2 is a copy of the seismogram of the Montana earthquake of August 18, $1959,\left(M=7.2 \pm, \Delta=10.8^{\circ}\right)$, written by the direct strain recorder of the Isabella strain seismograph. With this combination the trace amplitude is proportional to strain, which for plane waves is proportional to ground particle velocity divided by wave speed. This appears similar to conventional seismograms except for the permanent offset indicated by the white lines drawn on the print and the tidal strain variation. In addition to the direct strain recorder, the Isabella seismograph is equipped with a recorder operating in conjunction with a resistance capacitance band-pass filter which at the time of this earthquake had the circuit constants and transfer characteristic shown in figure 3 . This filter attenuates the response to the 12 hour tidal strains and to waves with periods less than 20 minutes as indicated in the figure. Moreover, for shorter periods up to about 3 minutes the filter acts as a time integrator. Consequently, in this period range the written response corresponds to the time integral of the strain (times a magnification constant), which for any given plane wave is proportional to the ground particle displacement divided by the phase velocity. Thus, if the displacement is given as

$$
u=F\left(t-\frac{x}{C}\right)
$$


where $x$ is the coordinate parallel to the direction of propagation and $C$ is the phase velocity, the strain $\epsilon$ is

$$
\epsilon=\frac{\partial u}{\partial x}=-\frac{1}{C} \frac{\partial u}{\partial t}
$$

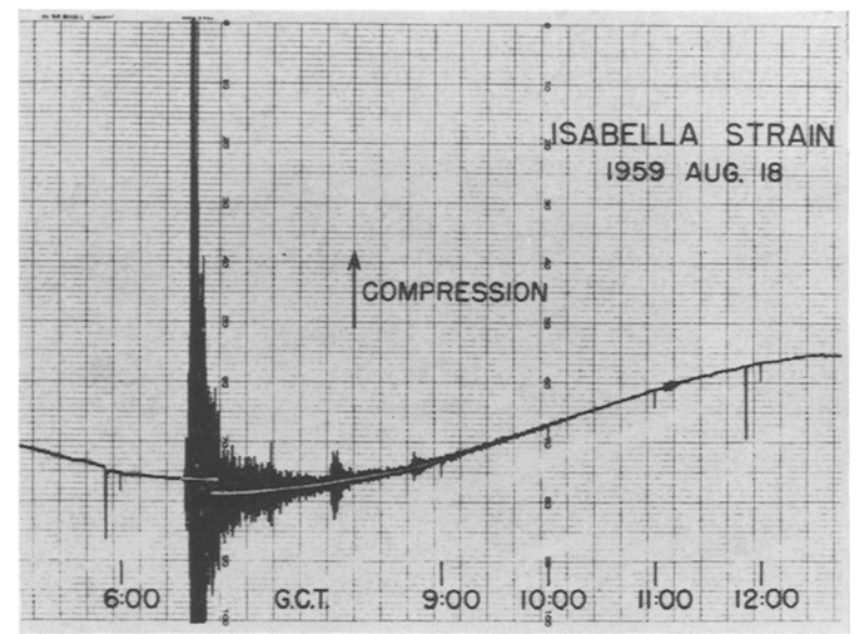

FIG. 2. Direct strain seismogram of Montana earthquake of August 19, 1959 made at Isabella, California.

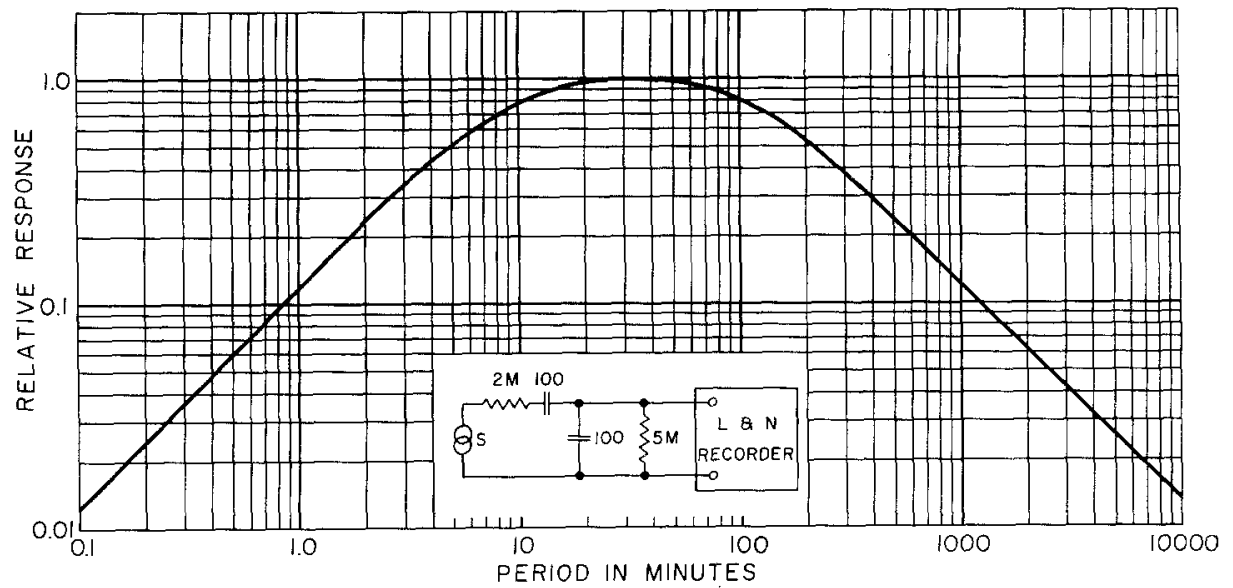

FIg. 3. Circuit and transfer characteristic of filter in use during earthquake of August 19, 1959.

and

$$
\int \epsilon d t=-\int \frac{1}{\bar{C}} \frac{\partial u}{\partial t} d t=-\frac{u}{\bar{C}}
$$

when $C$ is constant for a particular wave. A strain seismogram made with this filter thus differs from a recording of a true ground displacement meter in that the ampli- 
tudes $P, S$, and $R$ are each multiplied by the reciprocals of their respective surface phase velocities. The response of the strain seismograph with this filter for constant surface phase velocity is plotted in figure 4 in terms of ground displacement. A seismogram of the Montana earthquake of August 18, 1959 written with this com-

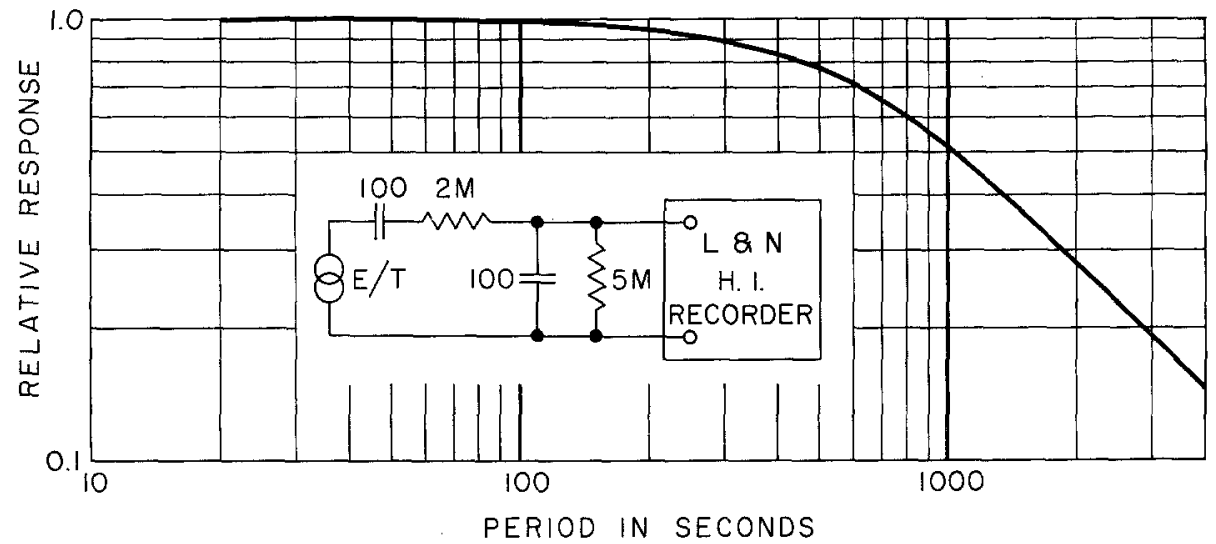

Frg. 4. Response of strain seismograph with indicated network in terms of ground particle displacement for constant phase velocity.

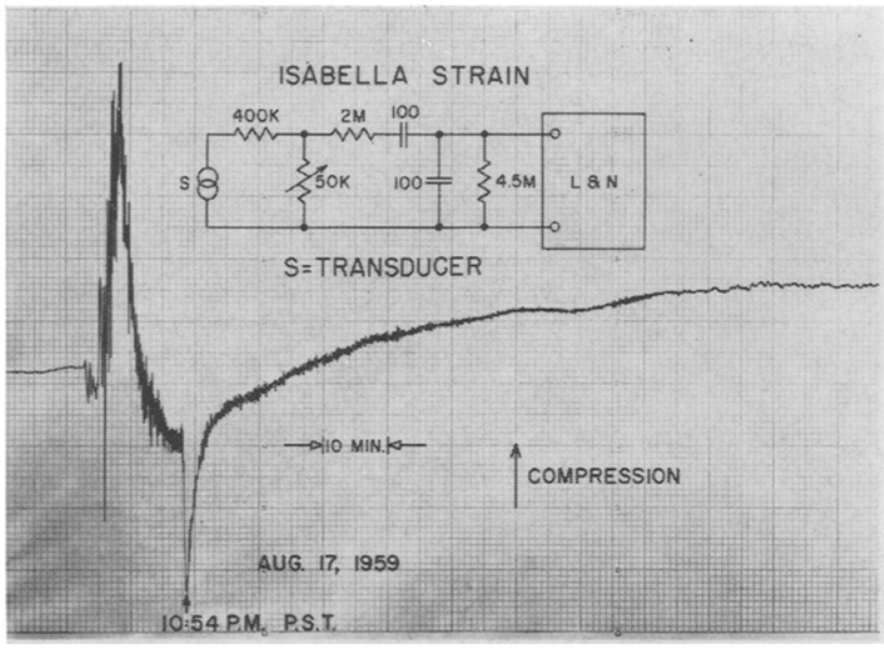

FIG. 5. Isabella strain seismogram of Montana earthquake of August 19, 1959 taken through indicated filter. The pulse at 10:45 PM is a calibration signal.

bination is reproduced in figure 5 . In figure 6 the Lamb pulse has been redrafted with the amplitudes of $P, S$, and $R$ multiplied respectively by the reciprocals of their velocities and with a reduced time scale to conform more nearly with the amplitude time scale relation of the seismogram of figure 5 shown in part on the right. It can be seen that in this seismogram the main features of the Lamb pulse response are 
reproduced. Superimposed on these are the attenuated short period oscillations and the network relaxation recovery coda of which the largest part was produced by the permanent dilatational offset of the ground. This seismogram thus shows that as seen from Isabella the source was a downward pulse in time. This is equivalent to a normal fault slip with the downward side facing Isabella. The field observations are in accordance with this result. It should be emphasized that not only does the earth medium depart from the homogeneous half-space assumed by Lamb but the actual

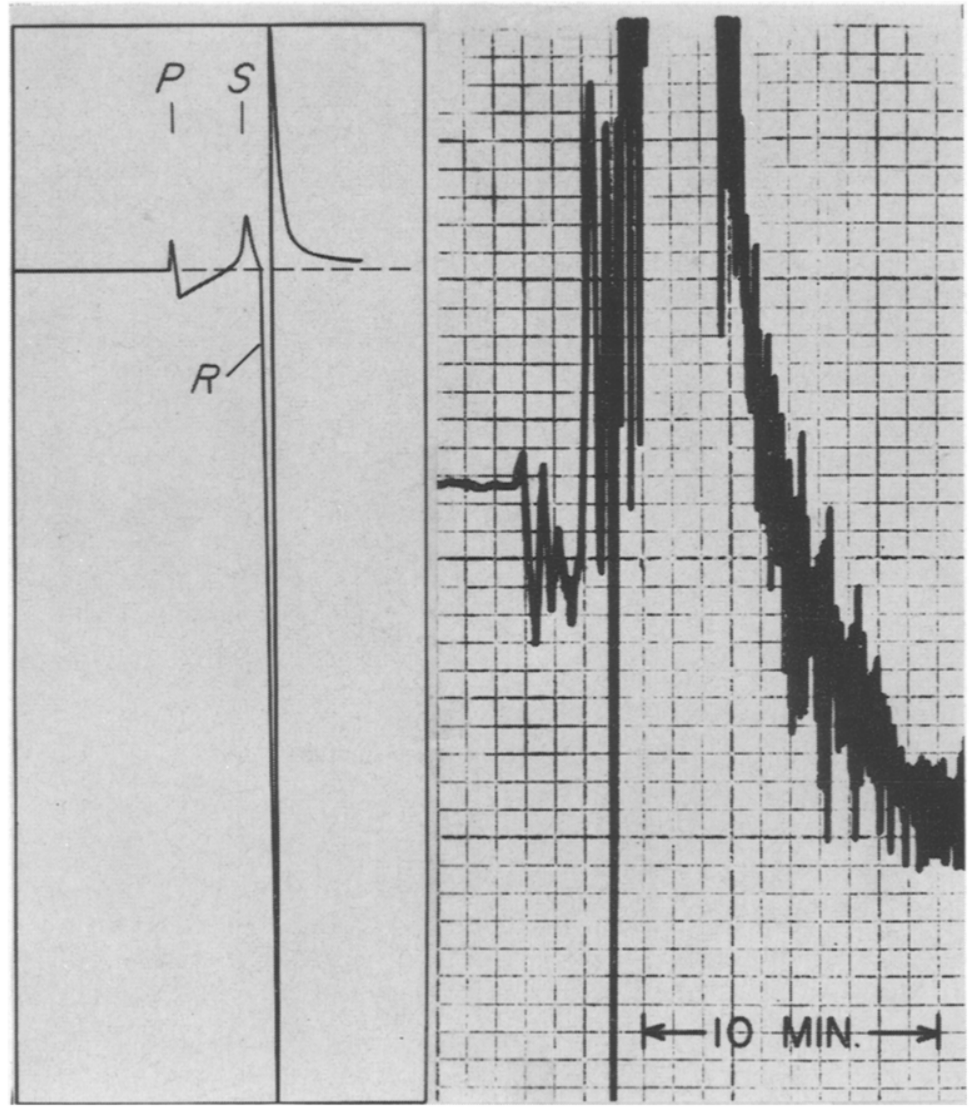

Frg. 6. Modified Lamb pulse pattern (left) with network strain seismogram of the Montana earthquake of August 19, 1959.

earthquake also differs from his point source in being extended in space and in following a function of time which differs from the form $S(t)=1 /\left(A+t^{2}\right)$ used by him. Consequently, one should not expect the observed and calculated patterns to be exactly alike. A third recorder in operation at Isabella at the time of the earthquake included a filter with two integration stages. A recording from this combination, together with the circuit is reproduced in figure 7. In this seismogram the shorter period oscillations are attenuated still further and the large initial dilatational pulse of the Rayleigh wave is very nearly obliterated. 


\section{The Peruvian Degp Earthquakes of August 1961}

In August 1961 a series of eight deep earthquakes $(h=600 \pm \mathrm{km}$ ) occurred in Peru with epicentral distances of about $620 \mathrm{~km}$ from the Naña strain seismograph installation. The two largest shocks magnitudes about 7 and $7 \frac{1}{2}$ were well recorded on the strain seismographs. They thus offered a unique observational opportunity to study large deep shocks having focal depths nearly equal to their epicentral distances. Table 1 gives the most recent data for three of the shocks kindly provided me by Mr. Leonard Murphy of the U.S.C. and G.S. plus an additional aftershock not appearing on the U.S.C. and G.S. cards.

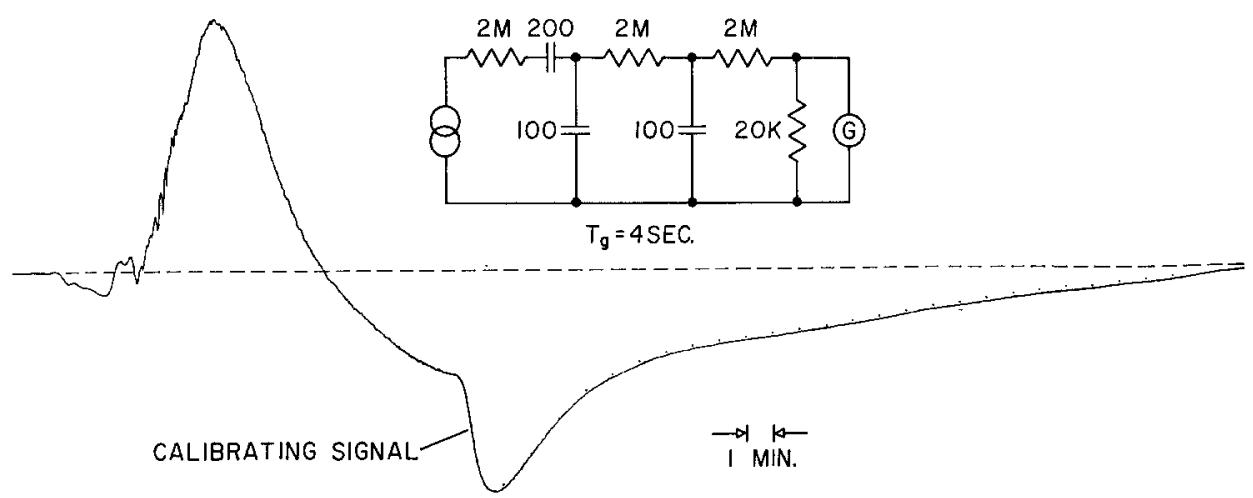

FIG. 7. Isabella strain seismogram of Montana earthquake of August 19, 1959 taken with two stage integrating filter.

TABLE I

Peruvian Defe Shocks 1961

\begin{tabular}{|c|c|c|c|c|c|}
\hline Shock No. & Date & Time & $\lambda$ & $\phi$ & $M$ \\
\hline 1 & 19 Aug. & $0509 \quad 49.5$ & $70.8^{\circ} \mathrm{W}$ & $10.8^{\circ} \mathrm{S}$ & 7 \\
\hline 2 & 19 Aug. & 0518 approx. & - & - & About 6 Aftershock \\
\hline 3 & 31 Aug. & 014838 & $70.9^{\circ}$ & $10.7^{\circ}$ & About 6.5 Foreshock \\
\hline 4 & 31 Aug. & 015707.9 & $70.6^{\circ}$ & $10.35^{\circ}$ & 7.5 \\
\hline
\end{tabular}

In addition to the direct strain recordings, the shocks were also recorded through the band-pass filter shown with its transfer characteristic in figure 8 . This filter was designed to provide maximum response for the lowest free period of the earth. Compared with the filter of figure 3 used earlier it has greater attenuation for the 12 hour tidal period and an increased time constant for the shorter period integration range.

Figure 9 is a copy of the seismogram of the earthquake of August 31, 1961 and a foreshock written with this filter on the Naña E $29^{\circ} 35^{\prime} \mathrm{N}$ component (marked E-W) strain seismograph. Aside from the small amplitude short period oscillations, this portion of the seismogram exhibits only $P$ and $S$ pulses owing to its small hypocentral distance. The trace amplitude is proportional to the time integral of the strain for periods up to about 20 minutes. A copy of a similar seismogram of the earth- 
quake of August 19, 1961 and an aftershock taken on the Naña E $29^{\circ} 3 \tilde{0}^{\prime} \mathrm{N}$ strain seismograph is shown on the left in figure 10. The epicentral distances of the August 19 and 31 shocks differ by only a fraction of a degree. Their recorded patterns are very nearly identical except for size. Since the Lamb calculations refer to surface sources it was necessary to compare these seismograms with the wave pattern predicted by Pekeris (1955) for a buried source. Figure 11 copied from Pekeris' paper shows the calculated surface radial displacement produced by a buried vertical downward point force in the form of a step in time for epicentral-depth ratios $r / h$ of 0.707 and 1.0 respectively. Although in these two earthquakes the actual ratio $r / h$ was nearer 1 , (assuming the correctness of the epicenter locations), the

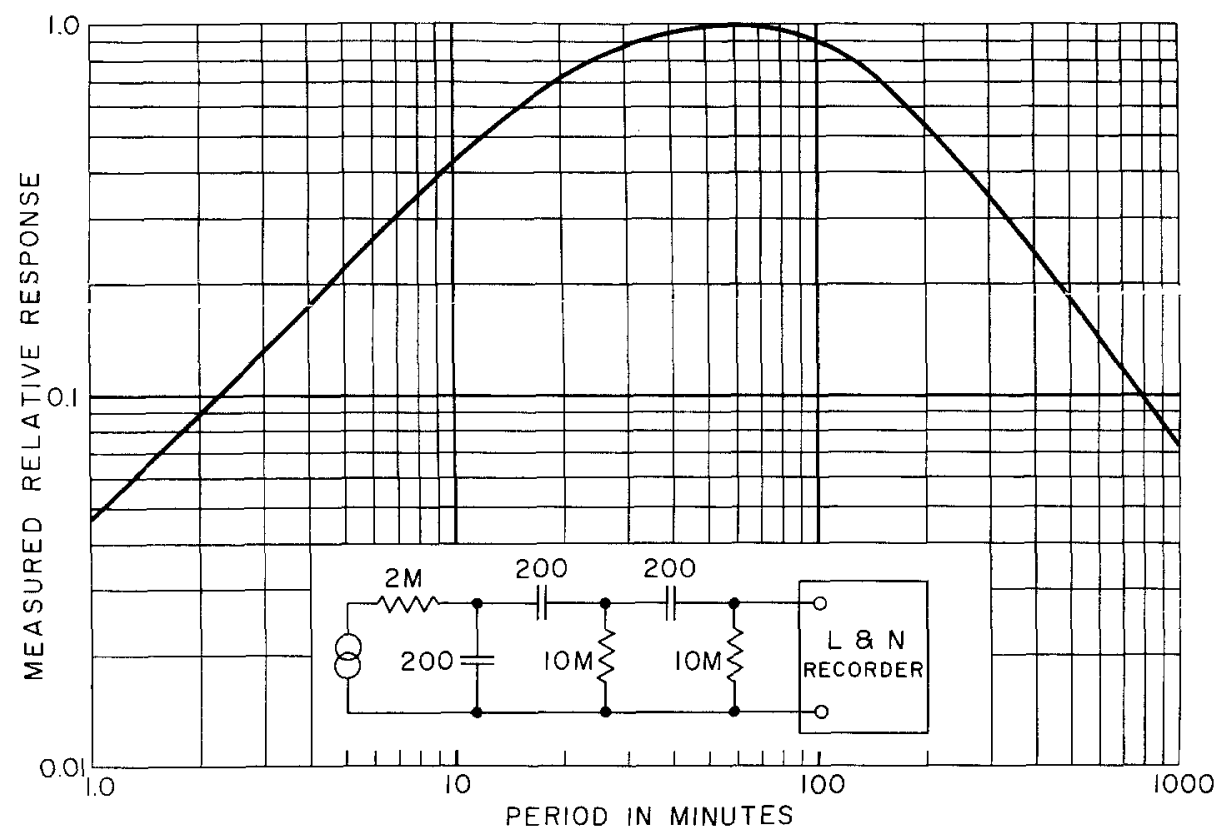

FIG. 8. Circuit and transfer characteristic of Ñaña filter network.

seismograms indicate that the 0.707 ratio provides the better fit. This divergence possibly results from departures of the actual medium from the assumed homogeneous half-space. On the right in figure 10 the pattern for the case $r / h=0.707$ has been redrafted making the amplitude of $S$ greater than that of $P$ in the ratio $C_{p} / C_{s}=$ 1.82 to correspond more nearly with the characteristics of the strain integral mentioned earlier. In addition, the time scale was compressed in relation to the amplitudes to correspond more nearly with the seismogram. Neglecting the small amplitude short period oscillations, the two seismograms of figures 9 and 10 resemble the computed pattern very closely. In these two deep earthquakes the wave patterns thus indicate a buried source in the form of a vertical downward force having the form of a step in time rather than an impulse as found in the case of the Montana earthquake. It is difficult to understand how a source of this kind could result from faulting unless the elastic moduli on the two sides of the fault differ so 
greatly that the rebound amplitude on one side is negligible in comparison with that on the other. The observational evidence is consistent with a source in the form of a sudden contraction of a volume of rock at the hypocenter such as might result from a sudden change of state. The radiation pattern from a source of this kind would depend in part on the speed with which the process of change is propagated within the active volume. If it is comparable with the wave speed all stations would record dilatations for the initial short period $p$ waves as shown at $\mathrm{A}$, figure 12 . The dashed

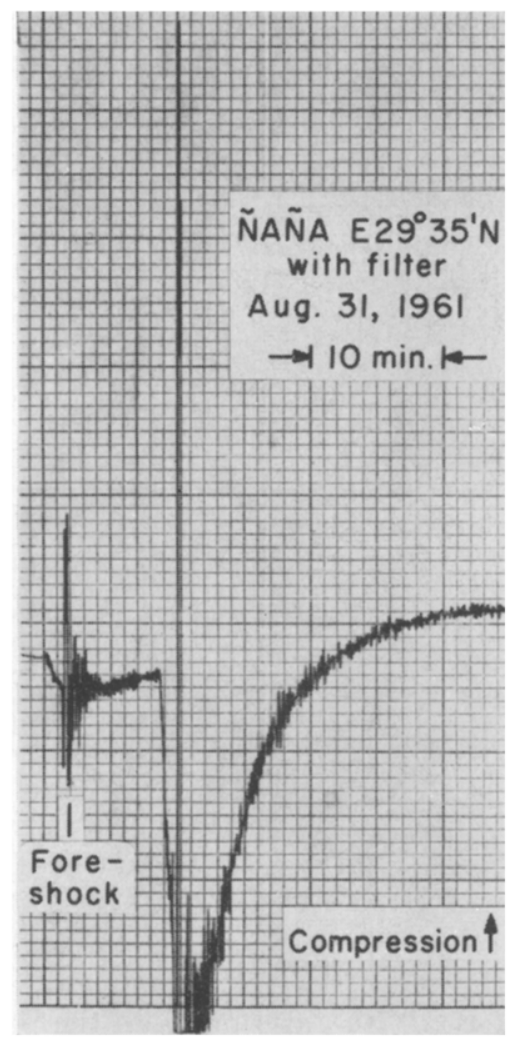

Frg. 9. Portion of seismogram of the deep earthquake of August 31, 1961 written with the filter of fig. 8 by the $\tilde{\mathrm{N}}$ aña $\mathrm{E} 29^{\circ} 35^{\prime} \mathrm{N}$ component strain seismograph.

lines represent the outline of the affected volume before the earthquake and the crosshatched square its initial contracted outline. The arrows indicate the directions of first motions of the original boundary in response to the hydrostatic pressure. Following the initial contraction, gravity would result in filling the void by a general downward movement of the overlying material and a downward movement of the contracted volume itself as represented at B, figure 12. Since the focal depth is 600 kilometers, the time required to complete the downward movement is of the order of $h / C_{p}=75$ seconds, where $C_{p}$ is the longitudinal wave velocity. Thus for wave periods longer than about a minute the contraction source is approximately equiva- 
Ient to the Pekeris step owing to the action of gravity. In a private communication, Father William Stauder indicated to me that a preliminary search indicated that these earthquakes produced initial $P$ wave dilatations at all stations and that $S$ waves were all polarized vertically. These preliminary observations are in accordance with a contraction source. On the assumption that these two earthquakes were generated

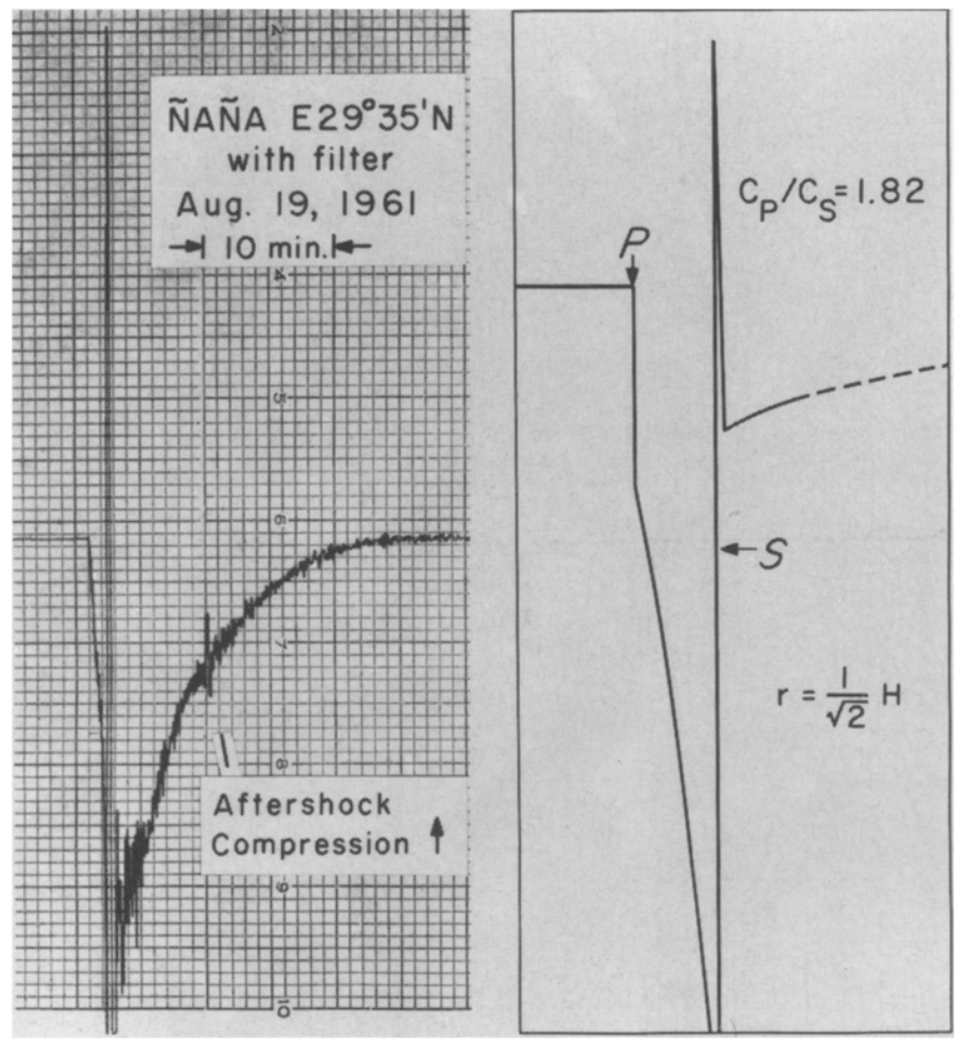

Fig. 10. Portion of seismogram of the earthquake of August 19, 1961 (on the left) written with the filter of fig. 8 by the Naña $E 29^{\circ} 35^{\prime} \mathrm{N}$ component strain seismograph. On the right the Pekeris buried source pattern for the case $r / h=0.707$ is shown redrafted to make the amplitude of $S$ greater than $P$ in the ratio $C_{p} / C_{6}=1.82$ and with a reduced time scale.

by volume contractions one may make some rough computations of the size of volume necessary to produce the required energy. If the volume contracts by an amount $\Delta V$, the energy involved in filling the resulting void by hydrostatic pressure is $E=$ $P \Delta V$ where $P$ is the hydrostatic pressure. Taking $E$ as $10^{23}$ ergs and $P$ as $2 \times 10^{11}$ dynes $/ \mathrm{cm}^{2}$, we have

$$
\Delta V=E / P=\frac{10^{23}}{2 \times 10^{11}}=5 \times 10^{11} \mathrm{~cm}^{3}=5 \times 10^{-4} \mathrm{~km}^{3} .
$$

Let us suppose that the fractional change of volume is $\Delta V / V_{0}=1 / 100$ where $V_{0}$ is the whole volume of rock which contracts. Then $V_{0}=100 \Delta V=5 \times 10^{-2} \mathrm{~km}^{3}$ which 
is a very small volume indeed. If this were a cube, its side is $V^{1 / 3}=\left(5 \times 10^{-2}\right)^{1 / 3}=$ $0.37 \mathrm{~km}$. The August 31 shock has an energy close to the maximum for earthquakes of its depth. Yet on the basis of the contraction mechanism the volume of the source is very much less than that associated with known faulting sources of similar energy.

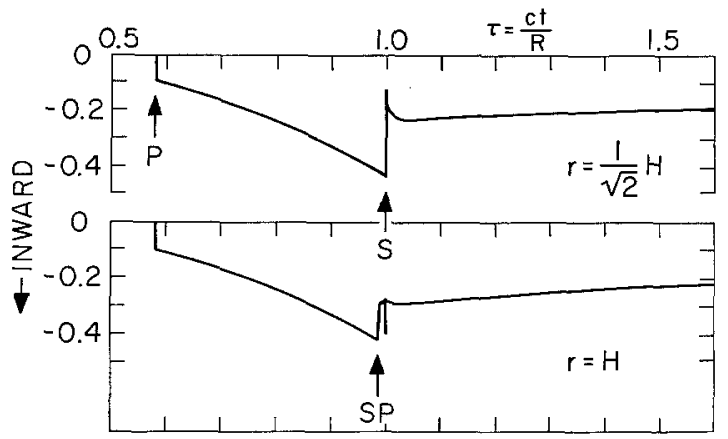

FIG. 11. Surface radial displacement produced by a buried step source for the cases $r=1 \sqrt{2}$ and $r=h$ as calculated by Pekeris.
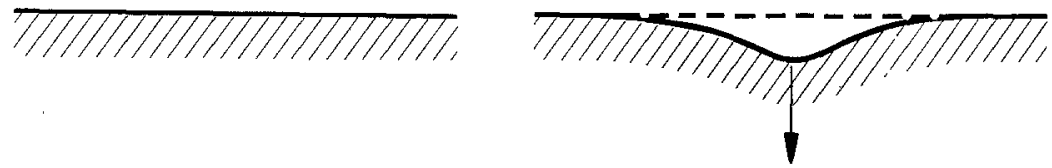

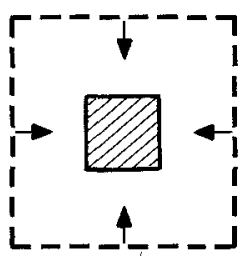

A

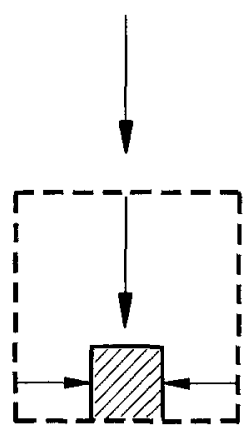

$\mathrm{B}$

FIG. 12. Contraction source. A initial motions. B final motions.

\section{ACKNOWLEDGMENT}

This research was supported by Contract AF-AFOSR-62-355 of the Air Force Office of Scientific Research as part of the Advanced Research Projects Agency's VELA UNIfokm Program.

\section{REFERENCES}

Benioff, Hugo

1959. "Fused Quartz Extensometer for Secular, Tidal and Seismic Strains," Geol. Soc. America Bull., V. 70, 1019-1032. 
Kaufman, S., and W. L. Roever

1951. "Laboratory Studies of Transient Elastic Waves", Proc. Third World Pet. Cong., Sec. 1, 538-545, Leiden.

Lamb, H.

1904. "The Propagation of Tremors Over the Surface of an Elastic Solid," Phil. Trans, Roy. Soc., Ser. A, 203, 1-42.

Pekeris, C. L.

1955. "The Seismic Buried Pulse," Proc. Nat. Acad. Sci., 41, No. 9, 629-639.

Tatel, Howard

1954. "Note on the Nature of a Seismogram-II," Jour. Geophys. Res., 59, No. 2, 289-294.

Seismologrcal Laboratory

Calffornia Institute of Technology

Pasadena, California

(Division of the Geological Sciences, Contribution No. 1163)

Manuscript received April 9, 1963. 